This work is licensed under a Creative Commons Attribution 4.0 International License. Ovaj rad dostupan je za upotrebu pod licencom Creative Commons Imenovanje 4.0 međunarodna.

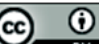

Nicolai GLASENAPP

Universität Koblenz-Landau, Deutschland Universitätsstraße 1, 56070 Koblenz

nglasenapp@uni-koblenz.de
Origineller wissenschaftlicher Beitrag

Original Research Article

Erhalten am 2. 7. 2021

Received: 2 July 2021

Angenommen am 19. 8. 2021

Accepted: 19 August 2021

\title{
LITERARISCHE ANAMNESEN. KRANKHEITEN UND IHRE TEXTLICHEN ANFÄNGE
}

\section{Zusammenfassung}

Literarische Texte etablieren Krankheiten als Komponenten einer kommunikativen Struktur, die Krankheit selbst konstituiert. Es ist zu überprüfen, ob Literatur an einem Prozess teilhaftig ist, der als Enttabuisierung von Krankheit bezeichnet werden kann oder ob die spezifische Konstruktion literarischer Texte zu Tabuisierungen beiträgt. Der vorliegende Beitrag untersucht Textbeispiele verschiedener Epochen und fragt nach dem Stellenwert ihrer Anfänge bei der Vorstellung von Krankheit. Dabei etabliert der Aufsatz zentrale Kategorien, die mit der Konfiguration von Krankheiten und ihren textlichen Anfängen verbunden sind: symbolische Räume; Kausalität und Schicksal; Handlungen als Krankheit; Krankheit als offene Wunde; Körpergrenzen und epistemische Schließungen; Setzung und Suspension von Krankheit als Teil von Interpretation. Als ein zentrales Ergebnis stellt der Beitrag heraus, dass Krankheit in den behandelten Beispielen vielfach nicht vollständig enttabuisiert wird, sondern vielmehr zwischen Tabuisierung und Enttabuisierung oszilliert. Auf diese Weise erfasst Literatur Krankheit umfassend und umgeht die Problematik, eine rationale Perspektive zu verabsolutieren, die wichtigen Aspekte menschlicher Existenz marginalisiert. Dies hat bedeutende Implikationen für die Themenfelder Literatur, Wissen und Krankheit.

Schlüsselwörter: Krankheit; (Ent-)Tabuisierung, Georg Büchner; Thomas Mann; Gottfried Benn; Franz Kafka; Georg Heym; Alfred Döblin; David Wagner; Wunde; Körpergrenzen 


\section{Einleitung}

Und jedem Anfang wohnt ein Zauber inne (Hermann Hesse: Stufen)

Hermann Hesses vielzitiertem Gedicht Stufen folgend birgt jeder literarische Text etwas Magisches, denn keiner kommt ohne einen Anfang aus. Peter-André Alts Betrachtungen zu Texten von weltliterarischem Rang und ihren ersten Sätzen weisen diese Komponente ebenfalls auf:

Am Beginn jeder Erzählung steht ein Verführungsversuch. Scheinbar aus dem Nichts kommt ein Satz, der seine Leser gewinnen und verstricken soll; er will Leser bezaubern, damit sie dem, was folgt, ihre Zeit opfern [...]. Deshalb formuliert ein erster Satz das lockende Angebot, den Pakt mit dem Buch zu schließen. Dieser Pakt verlangt den Lesern etwas ab, denn sie sollen sich dem Text ausliefern, ihm Zeit schenken und ihr Leben mit ihm teilen (Alt 10).

Bezüge zu Zauber und Magie scheinen auch in der Genese des Tabu-Begriffs auf (vgl. Trumm 12-13): Die Unberührbarkeit des Reinen und Heiligen und der Berührungsakt als Entweihung deuten an, was mit Krankheit als einer möglichen Sanktion einhergehen kann. Dass literarische Anfänge vielschichtig sind und damit einen eigenständigen Untersuchungsbereich eröffnen, wurde ebenso bemerkt wie die Relevanz des Endes als Gegenstück (vgl. Engelns/Löser/Nover). Auch Krankheiten folgen einem Muster, das auf einen Anfang rekurriert, wohingegen das Ende von Krankheit der Dichotomie Heilung oder Tod folgt (vgl. Glasenapp). Während im medizinischen Kontext der Beginn von Krankheit häufig mit einer ärztlichen Diagnose einhergeht, die Krankheit ex post Krankheit feststellt, folgen Krankheitsanfänge in literarischen Texten unterschiedlichen Gesetzmäßigkeiten: Vielfach liegt der Ausbruch von Krankheiten zunächst im Verborgenen. Damit ist nicht ausgeschlossen, dass eine erzählende Instanz explizite Hinweise gibt oder über ein symbolisches Gefüge entsprechende Andeutungen vorgenommen werden - dies begegnet Lesenden etwa in Texten von Thomas Mann wie Buddenbrooks. Verfall einer Familie, Der Tod in Venedig oder Doktor Faustus. Das Leben des deutschen Tonsetzers Adrian Leverkühn.

Einen ebenfalls symbolischen, aber wesentlich eruptiveren Krankheitsanfang setzt David Wagners Text Leben: Der Ich-Erzähler erblickt plötzlich sein 
eigenes Blut in der Badewanne. Die Unmittelbarkeit und Dinglichkeit der Darstellung hält dabei Bewusstwerdung von Krankheit und Ungewissheit in einer kombinatorischen Schwebe und beschreibt einen Übergangszustand. Die Explizitheit der Darstellung bedingt eine Enttabuisierung, hält aber ein Stück des Krankheitstabus aufrecht.

Die bewusste Reflexion und Versprachlichung von Krankheit im literarischen Text zwingen dazu, Krankheit der Aura des Tabuisierten zu entkleiden. Dennoch - so eine zentrale arbeitsleitende These für den vorliegenden Beitrag - ist die literarische Inszenierung von Krankheitsanfängen stets in einem Zwischenraum situiert, wenn mit Krankheit die Ränder des Aussprech- und Erklärbaren umkreist werden. Es erfolgt immer nur eine Annäherung an das Schicksal Krankheit und gerade psychische Krankheitsdispositionen begründen ein Paradoxon des zugleich Beschreibungswürdigen und Nichtbeschreibbaren.

Diesen Auffälligkeiten soll anhand signifikanter Textbeispiele aus verschiedenen literaturgeschichtlichen Epochen nachgegangen werden. In der Analyse von literarischen Krankheitsanfängen kommt ein Vorgang der Transgression zur Betrachtung, bei dem der literarische Text den Übergang von unbewusster Krankheit zu ihrer Bewusstwerdung inszeniert und somit prozesshaft eine Bewegung vollzieht (vgl. Clare et al.). Krankheit fungiert in diesem Sinne auch als perspektivische Grundlage, um literarische Texte darin zu untersuchen, wie sie die Grenzen von Sagbarkeit und Unsagbarkeit ausloten (vgl. Habermann) und bietet das Potenzial, literarische Kommunikation auf einer Metaebene zu reflektieren, die einen Topos der Literaturgeschichte fortschreibt, der mit der Sprachskepsis um 1900 und dem Brief des Lord Chandos von Hugo Hofmannsthal einen Höhepunkt zu verzeichnen hat.

Der vorliegende Beitrag nähert sich einer Problematik, die in einem wesentlichen Zusammenhang mit Krankheit steht. Dabei wird vor allem eine strukturalistisch grundierte Betrachtungsweise mit einem Schwerpunkt auf der Narration der Texte gewählt, wenn der kommunikative Beginn von Krankheiten in literarischen Texten untersucht und danach gefragt wird, ob sie beispielsweise explizit oder implizit thematisiert werden und inwieweit damit eine Enttabuisierung oder Tabuisierung einhergeht. Zudem wird eine epistemologische Fragestellung in Verbindung mit Krankheit berührt (vgl. Shattuck): Welches Wissen über Krankheit rückt in den Vordergrund, welche Momente der Aussparung oder Verdrängung sind zu identifizieren und was sagen entsprechende Beob- 
achtungen über ,jene Differenz von ,krank' und ,gesund', mit der Gesellschaften immer auch einen Gradmesser ihrer eigenen Stabilität festlegen“? (Erhart 2). Unter diesen Prämissen steht Tabuisierung auch einer Verschleierung nahe, welche von Michel Foucault als ein Charakteristikum von Diskursen angeführt wird und ihre Wirksamkeit im Unsichtbaren sichert (vgl. Foucault 16-17).

Am Ende wird zu eruieren sein, ob Krankheitsanfänge in literarischen Texten zu einer Verzauberung oder Entzauberung tendieren, die Tabus aufrechterhalten oder auflösen. Möglicherweise oszilliert die Darstellung von Krankheit zwischen Tabu und Enttabuisierung und bleibt insofern ambivalent, ohne mit einem der beiden beschriebenen Pole dezidiert kongruent zu sein. Im folgenden Kapitel sollen einige grundsätzliche Überlegungen zur Strukturierung der Analyse vorgenommen worden. Die entworfene Heuristik dient der Orientierung und Charakterisierung wiederkehrender Muster innerhalb der untersuchten literarischen Texte.

\section{Strukturelle Grundlagen}

Selten entspricht der ärztliche Befund von Krankheit bei literarischen Texten so sehr dem Anfang, wie es bei Hilda Röders Roman Henning flieht vor dem Vergessen. Ein Roman über Würde, Alzheimer-Erkrankung und Sterbehilfe aus dem Jahr 2013 der Fall ist: Die Handlung beginnt mit einem Arztgespräch und der Diagnose Alzheimer-Demenz. Zunächst leugnet der Protagonist Henning Landes, dass er vom Krankheitsbild der Alzheimer-Demenz betroffen sein könnte. In der Folge häufen sich aber Erlebnisse, die den Befund bestätigen. Strukturell fällt damit die Diagnose mit dem Beginn der Krankheit selbst zusammen. So entsteht bei der Lektüre der seltsame Eindruck, als schaffe erst die Diagnose die Realität einer Krankheit, obwohl ein medizinischer Befund der Existenz von Krankheit eigentlich immer nur nachgeordnet sein und bestätigen kann, was latent bereits existent war.

Wesentlich häufiger umkreisen literarische Texte die Krankheit von Figuren und evozieren ein Spiel aus Andeutung und Verschleierung, während die konkrete Benennung diesen Prozess unterbricht, aussetzt und schlussendlich Krankheit selbst trivialisiert, indem sie auf Begriffe gebracht und dadurch reduziert wird - es scheint, als sei Krankheit damit sprachlich gebannt. Dabei handelt es sich um einen Irrglauben. Denn die anthropologische Herausforderung, die in Krankheiten selbst liegt, sichert einen Überschuss des Ungeklärten, 
der als Rest bestehen bleibt und in verschiedenen Krankheitsbildern mit unterschiedlichen Konstellationen wiederkehrt und neue Begriffe hervorbringt wie erfordert. Daran lässt sich ablesen, dass es sich bei der Frage nach dem Anfang von Krankheit in einem literarischen Text um eine vielschichtige und plurale Angelegenheit handelt.

Benennung von Krankheit folgt einer Tradition, die auf die magische Funktion sprachlicher Akte rekurriert: Durch das bloße Benennen wird identifiziert und damit auf eine Wissensgrundlage Bezug genommen, die suggeriert, es sei bekannt und gewiss, worum es sich beim Benannten handelt. Insofern ist ein einzelner Begriff bereits imstande, einen ganzen medizinischen Wissenskomplex aufzurufen. Reziprok führt ein Mangel an Wissens- und Forschungsgrundlagen zu Krankheiten und zu einer Vagheit, die durch Metaphern ausgeglichen werden kann - sie tragen dann über den medizinischen Kontext hinaus zu einer Semantisierung von Krankheit bei, die sich in gesellschaftlichen und kulturellen Vorstellungen manifestiert. Ein anderes Phänomen liegt vor, wenn die Benennung von Krankheit bewusst ausgespart wird, um damit gerade nicht aufzurufen, was an mit der Krankheit zu assoziierenden Implikationen bekannt und präsent ist (vgl. Schwahl/Thienel 261-62). Somit kommen der Erwähnung und Benennung selbst unterschiedliche Funktionen zu, die spezifisch zu betrachten sind und sich an den kommunikativen Eigenheiten des Einzeltextes zu orientieren haben. Daraus lässt sich die Frage ableiten: Wie werden Krankheiten sprachlich in Texten eingeführt und welche Semantisierungen sind damit verbunden? Zeichnet sich dieser Vorgang durch eine Ent- oder Verschleierung aus?

Von Krankheitsanfängen auszugehen, impliziert eine zeitliche Struktur. Dabei werden auch narratologische Aspekte berührt: Was wem wie bekannt ist und wann wem wie erzählt wird, hat zur Folge, dass ein Wissen über Krankheit bekannt wird oder unbekannt bleibt. Zudem wird dadurch ein wesentlicher Unterschied markiert, ob eine Erzählinstanz Hinweise liefert, die Lesenden, aber nicht betroffenen Figuren bekannt sind oder ob mit Krankheit verbundene Vorgänge als Selbsterkenntnisprozesse von Figuren inszeniert werden. Derartige Aspekte gilt es im Blick zu behalten, um Krankheitsanfängen in literarischen Texten einen angemessenen Status zuzuordnen. 


\section{Raumsymbolik (Georg Büchners Lenz, Thomas Manns Buddenbrooks. Verfall einer Familie und Der Tod in Venedig)}

Der Beginn von Georg Büchners Lenz erscheint unauffällig. Dem Gang durch das Gebirge und der Landschaftsbeschreibung haftet nichts an, das intuitiv als außergewöhnlich zu charakterisieren wäre, auch wenn mit dieser Beschreibung bereits eine Stimmung zu assoziieren ist, die von der Natur auf menschliche Empfindungen schließen lassen könnte und beispielsweise in der doppelten Erwähnung von Schwere zum Ausdruck kommt. Eine nähere Charakterisierung des Protagonisten verbindet sich erst mit den folgenden Textaussagen: „Er ging gleichgültig weiter, es lag ihm nichts am Weg, bald auf- bald abwärts. Müdigkeit spürte er keine, nur war es ihm manchmal unangenehm, daß er nicht auf dem Kopf gehen konnte“ (Büchner 137).

Die zitierte Passage suggeriert eine Unabhängigkeit zwischen dargestelltem Mensch und Landschaft, die auf Distanz schließen lassen könnte. Tatsächlich wird Lenz aber als Teil dieser Landschaft eingeführt, wenn das erste, was Lesende von ihm erfahren, seinen Gang durch diese Landschaft betrifft. Und die folgenden Zeilen offenbaren, dass dieses Subjekt sehr wohl in einer Auseinandersetzung mit der räumlichen Umgebung steht, die zugleich Auseinandersetzung mit sich selbst ist (vgl. Stiening 578-79):

Anfangs drängte es ihm in der Brust, wenn das Gestein so wegsprang, der graue Wald sich unter ihm schüttelte, und der Nebel die Formen bald verschlang, bald die gewaltigen Glieder halb enthüllte; es drängte in ihm, er suche nach etwas, wie nach verlorenen Träumen, aber er fand nichts. (Büchner 137).

Einmal mehr fällt die sprachliche Wiederholung - hier des inneren Drängens - auf und es liegt nahe, im Textbeginn die Eröffnung eines symbolischen Raumes zu sehen. Denn tatsächlich wird die Landschaft von Lenz erlebt und sie erfährt auf Grundlage seiner Wahrnehmung Gestaltung, die beispielsweise in wiederkehrenden Personifikationen erkennbar wird.

Die Verknüpfung von Krankheit mit einer Symbolik des Raumes erfolgt unter spezifischen kommunikativen Bedingungen: Sie macht anschaulich und lässt innere Dispositionen hervortreten, ist aber immer auch Projektionsfläche für dieses Innere. Dieser Doppelcharakter führt an krankheitsbedingte Wahrnehmung heran, bleibt dabei aber vielfach andeutend und präsentiert Wahr- 
nehmung als Komplex, der mit seinen Gegenständen verschmilzt. Mit Symbolik und Metaphorik wird Krankheit auf Grundlage eines uneigentlichen Sprechens nachvollziehbar gemacht. Es bleibt aber ein Rest, der sich dem Erklärenden entzieht und dementsprechend etwas, das dem Tabu eignet.

Der Symbolik des Naturraumes in Büchners Lenz steht eine Symbolik des Wohnraumes gegenüber (Reidy 27-28), wie sie bereits im Biedermeier, dann im Realismus eines Theodor Fontane oder auch im Buddenbrooks-Roman von Thomas Mann vorzufinden ist. Zu Beginn des Letztgenannten scheint es nicht, als könne der Text etwas mit dem Thema Krankheit zu tun haben: Die opulente Familienfeier, die den Text einleitet, erscheint geprägt von Wohlstand und Lebensfreude. Bei einer aufmerksamen und wiederholten Lektüre werden jedoch erste Spuren des Verfalls erkennbar, den der Untertitel explizit benennt: Getränke und Speisen erweisen sich als besonders schwer und bewirken beim jungen Christian Buddenbrook eine ausgeprägte Magenverstimmung. Und auch die Gestaltung der prunkvollen Inneneinrichtung deutet die Möglichkeit an, dass die vordergründig harmonische familiäre Ordnung nicht über alle Zweifel erhaben bleiben wird. Eine erste Erwähnung von Jean Buddenbrooks Nervosität antizipiert jenen Zug zur Sensibilität, der sich in der Kränklichkeit Hannos sowie der Neigung zur Kunst niederschlagen und damit das Schicksal der Familie besiegeln wird. Sie steht der Robustheit entgegen, die noch bei Johann Buddenbrook, dem Älteren Fundament für Geschäftstüchtigkeit und Familienordnung ist. Mag es sich bei Buddenbrooks um ganz andere Vorzeichen des Räumlichen als bei Lenz handeln, so bleibt doch eine Verbindung des symbolischen Raumes mit Krankheit erhalten, die mehr Andeutung als Enttabuisierung impliziert.

Mit der Schriftstellerfigur Gustav von Aschenbach in Der Tod in Venedig entwirft Mann einen weiteren Protagonisten, an dem schon früh im Text bemerkt wird, dass er „überreizt“ sei (Mann 9). Er versucht diesem Zustand anfangs durch Bewegung im Freien beizukommen. Damit ergeben sich Parallelen zu der Lenz-Figur und den Familienmitgliedern der Buddenbrooks. Die Bewegung durch den symbolischen Raum Venedig und die später zum Tod führende Infektion mit Cholera figuriert ebenfalls einen Zusammenhang zwischen Raum und Krankheit, dessen erste Anzeichen schon mit dem Textanfang initiiert und von ihm im Rahmen der Narration als folgerichtige, weil final motivierte Entwicklung vermittelt werden (vgl. Martínez/Scheffel 118-119). Eine zusätzliche Dimension der Tabuisierung und Enttabuisierung entsteht durch Aschenbachs Zuneigung gegenüber dem jungen Tadzio, die beispielsweise im Symptom des 
Schwindels körperlichen Ausdruck erfährt (vgl. Müller-Tamm 706). Krankheit steht dadurch weniger im Zentrum einer Tabuisierung, sondern wird zu einem Katalysator für Aschenbachs enthemmte Leidenschaften und trägt zu einer Enttabuisierung bei (vgl. Shi 111-12).

\section{Kausalität und Schicksal (Thomas Manns Der kleine Herr Friedemann und Gottfried Benns Gehirne)}

Unter epistemologischen Gesichtspunkten spielt die Genese von Krankheit eine wichtige Rolle. Damit rücken Fragen nach Ursprüngen und Kausalitäten in das Zentrum des Interesses. Der Anfang von Thomas Manns Der kleine Herr Friedemann und der Einstieg in Gottfried Benns Novellen-Zyklus Gehirne verdeutlichen eine Suche nach Erklärungen für Krankheit innerhalb literarischer Texte. Mit „Die Amme hatte die Schuld“ eröffnet Manns Text und deutet die eine Kausalität an (Mann, Der kleine Herr 66): Die anschließend geschilderte Abfolge von Vorgängen, welche zur Verkrüppelung und - den Ausführungen der Erzählinstanz folgend - ,Unförmigkeit' von Johannes Friedemann führen, wird an die Handlungen einer Einzelperson rückgebunden, der unmittelbar die Schuld am Resultat zugesprochen werden kann. In nuce vollzieht der Text zu Beginn, was noch ausschweifender und weitreichender für Manns Romane gilt, wenn er nur allmählich wesentliche Entstehungszusammenhänge erläutert und entfaltet, worin die Schuld der Amme eigentlich besteht.

Der lakonische erste Satz wirkt in seiner Erklärung beinahe zu monokausal und der Text liefert dem Zweifel daran weitere Grundlagen: Die Mutter Johannes Friedemanns erkennt den Alkoholismus der Amme und statt ihr zu kündigen, gibt sie „ihr außer dem nahrhaften Bier ein Glas Rotwein täglich“ (ebd.). Der erste Satz spart also die Verstrickungen und unzureichenden Entscheidungen aus, die dem tragischen Unfall vorausgehen und erscheint wie ein Teil eines Ablenkungsmanövers, wäre er nicht vor allem dazu angetan, überhaupt einen Anlass für die folgenden Erklärungen zu liefern. Nicht zuletzt ist der Anfangssatz wie so oft bei Thomas Mann ironisch zu lesen - dann kann er als fehlendes Eingeständnis der Konsulin Friedemann verstanden werden, deren Handeln die Versehrung ihres Sohnes begünstigt, und markiert eine Leerstelle, denn die Setzung der Erklärung bedeutet zugleich die Zurücknahme anderer Möglichkeiten.

Es ist festzuhalten, dass am Anfang des Textes zwar die körperliche Erkran-

kung des titelgebenden Protagonisten in ihrer Entstehung beschrieben wird, 
mit dem Alkoholismus der Amme aber zusätzlich eine Krankheit eingeführt wird, die wiederum wesentlich dafür ist, dass eine weitere Erkrankung hervorgebracht wird - charakteristisch ist ein tabuisierender Umgang mit dem Alkoholismus der Amme, denn die damit einhergehende Problematik wird nicht etwa durch Konfrontation oder direkte Kündigung aufgelöst, sondern in Kauf genommen und durch die Beigabe von Spirituosen noch aufrecht erhalten.

Allein diese Feststellung reicht aus, um Krankheit eine besondere Präsenz zum Textbeginn zu attestieren. Jedoch kommt noch eine weitere Dimension hinzu: Nach dem Sturz von Johannes Friedemann geht seine Mutter nicht davon aus, dass er überleben werde. Die damit verbundene Haltung rührt vom Tod ihres Mannes, der „,von einer ebenso plötzlichen wie heftigen Krankheit dahingerafft wurde" (ebd.). Allein die ersten Zeilen des Textes verschaffen Krankheit also eine Omnipräsenz, die über eine Kausalität ihrer Entstehung auf Basis rationaler Erklärungsmuster hinausweist. Es kommt nicht von ungefähr, dass der Sturz von Johannes Friedemann als „Unglück“ (ebd.) bezeichnet wird. Die Schicksalshaftigkeit der Ereignisse entrückt sie dem Bereich nachvollziehbarer Kausalzusammenhänge.

Gestützt wird diese Charakteristik beispielsweise durch die wiederholte rhetorische Frage „Was half es [...]“ zu Beginn des Textes, die als erzählerischer Kommentar fungiert (ebd.). Und auch der konkrete Sturz Johannes Friedemanns wird in seinen Konsequenzen erwähnt, aber sein eigentlicher Ablauf wird ausgespart - stattdessen wird er als unwiderrufliches Faktum inszeniert, welches die Konsulin Friedemann und ihre drei Töchter bei ihrer Wiederankunft zuhause zur Kenntnis zu nehmen haben. Der Text entwirft für Krankheit also sowohl eine kausale als auch eine fatalistische Perspektive und liefert gleichermaßen Erklärungen wie Aussparungen. Beispielsweise bleibt die Krankheit des Vaters von Johannes Friedemann schimärenhaft und lässt kaum Schlüsse darauf zu, worum es sich eigentlich gehandelt haben mag. Diese Doppelperspektive auf Krankheit steht sinnbildlich für die Modernität von Manns Schreibweise, die sich bereits in diesem frühen Text zeigt. Durch sie entsteht ein Nebeneinander von Tabuisierung und Enttabuisierung.

Auch bei Benns Gehirne suggeriert der textliche Anfang Nachvollziehbarkeit von Dispositionen, die mit Krankheit einhergehen:

Rönne, ein junger Arzt, der früher viel seziert hatte, fuhr im Sommer vorigen Jahres durch Süddeutschland dem Norden zu. Er hatte die letzten 
Monate tatenlos verbracht; er war zwei Jahre lang an einem pathologischen Institut angestellt gewesen, das bedeutet, es waren ungefähr zweitausend Leichen ohne Besinnen durch seine Hände gegangen, und das hatte ihn in einer merkwürdigen und ungeklärten Weise erschöpft (Benn 19).

Der Text führt eine Konstellation aus Tätigkeit, Erschöpfung und Untätigkeit ein, um den Zustand Rönnes zu beschreiben. Anders als bei Manns Der kleine Herr Friedemann rückt damit die Psyche des Protagonisten in das Zentrum der Aufmerksamkeit. Seine Reise, die vage innerhalb von Deutschland verortet wird, deutet bereits einen Moment der Selbstfindung an. Bestätigt wird dieser Befund etwa auch durch das Vorhaben, die eigenen Lebenseindrücke schriftlich zu fixieren und zu reflektieren: „Ich will mir ein Buch kaufen und einen Stift; ich will mir jetzt möglichst vieles aufschreiben, damit nicht alles so herunterfließt. So viele Jahre lebte ich, und alles ist versunken“ (ebd.). Offen lässt der Beginn, ob die Besinnungslosigkeit, die zu Rönnes Lage führt, auf die toten Menschen oder ausgeführte Arbeitsvorgänge rekurriert. Fakt ist, dass seine berufliche Tätigkeit der Ausgangspunkt dafür ist. Die damit gelieferte Erklärung wird jedoch dadurch suspendiert, dass seine Erschöpfung rätselhaft bleibt.

Ähnlich wie bei Manns Der kleine Herr Friedemann kommt zum Ansatz der Plausibilisierung von Zuständen die Einwirkung einer Schicksalshaftigkeit: „Das Leben ist so allmächtig, dachte er, diese Hand wird es nicht unterwühlen können, und sah seine Rechte an" (ebd.). Zwar wird dieser omnipotente Einfluss nicht an eine personalisierte Instanz gebunden, sondern sachlich an das Leben als solches, doch wird damit eine Dimension angedacht, die über das eigene Handeln hinausweist und auf das einzelne Individuum einwirkt (vgl. Reents 131-32). Dieses Moment eines hereinbrechenden und unkontrollierbaren Lebens findet sich auch in den weiteren Novellen über Rönne, beispielsweise, wenn sein Kind erkrankt (vgl. Benn 29). Gerade der Beruf des Arztes und jene Faktoren, die ihn tagtäglich beeinflussen, eröffnen einen Reflexionsraum für existenzielle Fragen, die neben der Logik und Vernunft der eigenen Arbeit ihre Berechtigung haben. Der Text liefert eine einfache Erklärung für Rönnes krankheitsähnliche Symptome, schafft aber auch Momente, in denen Krankheit als Fatum dem reflektierenden Zugang entzogen wird und hält damit einen Schwebezustand aufrecht, der Enttabuisierung und Tabuisierung zugleich andeutet. 


\section{Handlungen als Krankheit (Georg Heyms Der Irre und Alfred Döblins Ermordung einer Butterblume)}

Schon die Titel von Georg Heyms Der Irre und Alfred Döblins Die Ermordung einer Butterblume suggerieren eine Abweichung von Normalität und rufen Muster psychischer Krankheit auf. Während Heym mit dem Titel einen Typus entwirft, manifestiert sich die Normabweichung bei Döblin durch den Hinweis auf eine Handlung, die auf eine krankheitsbedingte Wahrnehmungsdisposition hindeutet. Wie schon bei Büchners Lenz und Manns Der Tod in Venedig ist die Bewegung durch den Raum zentral - doch gerade die Art und Weise der Bewegung und die narrative Begleitung mit ihren Hinweisen auf die psychischen Zustände beider Protagonisten bilden einen Komplex, der genauerer Betrachtung bedarf.

Der Beginn von Heyms Text lässt annehmen, es handle sich um eine Entlassung, wenn ein Wärter dem Geschehen beiwohnt. Im ersten Absatz werden jedoch ausschließlich äußere Vorgänge beschrieben. Eine Zäsur erfolgt mit dem Satz: „So, und nun sollte die Welt etwas erleben“, der erstmals mit seiner Gedankenwelt das Innere des Protagonisten offenbart (Heym 18). Auch er geht in die entworfene Landschaft ein, wenn er sich in ein Blumenfeld mit Mohn und Schierling stürzt, bevor die Repräsentation seiner Gedanken fortgesetzt wird:

Er war also frei. Es war aber auch höchste Zeit, daß sie ihn herausgelassen hatten, denn sonst hätte er alle umgebracht, alle miteinander. Den dicken Direktor, den hätte er an seinem roten Spitzbart gekriegt und ihn unter die Wurstmaschine gezogen. Und der Assistenzarzt, dieses bucklige Schwein, dem hätte er noch mal das Gehirn zertreten. (ebd.)

Gewaltphantasien und die Tendenz zu abwegig erscheinenden Handlungen kennzeichnen den Irren. Dabei wird deutliche Kritik an der Einrichtung geübt, die ihn noch zuvor beherbergt hatte: „Und die Wärter in ihren weiß gestreiften Kitteln, die aussahen wie eine Bande Zuchthäusler, diese Schufte, die die Männer bestahlen und die Frauen auf den Klosetts vergewaltigen. Das war ja rein zum Verrücktwerden“ (ebd.). Der Text lässt offen, ob der Einfluss der Institution erst maßgeblich für das Bedürfnis des Irren nach Gewalt ist oder ob seine Neigung zu Menschenverachtung und Gewalt der Grund für seine Inhaftierung waren. Es zeigt sich eine „bewusst uneindeutig gehaltene Beziehung zwischen den Zwangsgedanken und -handlungen und der Ursache dieser Symptomatik“ (Kyora 871). Daran lässt sich ein Bruch mit der Konzeption des Irren als Typus 
erkennen, denn abweichend von einer schematisierten Darstellung wird er mit einem Reflexionsvermögen ausgestattet, das ihn dazu befähigt, die Resultate institutionalisierten Machtmissbrauchs zu erkennen. Der Text verhandelt also als ein zentrales Thema die Frage nach Normalität und hinterfragt damit verbundene Grenzziehungen. Die Figur des Irren zeichnet sich dabei selbst durch Entgrenzung aus, was sich vor allem in ihren - vollzogenen oder imaginierten - Handlungen abbildet. Mit der Tendenz zu einer Vereinigung mit der Natur mittels der Pflanzen- oder Tierwelt kommt es zu einem antizivilisatorischen Moment, das den Zuständen in der bewohnten Einrichtung entspricht und damit insgesamt „die Erkenntnis von einer deformierten Welt, eine Einsicht, die das Absonderliche als ihre realen Tatbestände versteht", präsent werden lässt (Schünemann 96).

In Döblins Die Ermordung einer Butterblume weisen die zum Teil manischen Handlungen von Michael Fischer auf eine vergleichbare Entgrenzungserfahrung hin, die als Resultat seiner Erfahrungen in der Stadt- und Berufswelt interpretiert werden kann. Zu Beginn zählt Fischer seine Schritte, bewegt sich unstet und vergisst seine Handlungen anschließend wieder. Zudem verrät seine Körpersprache eine psychische und emotionale Auseinandersetzung, deren eigentlicher Angriffspunkt mysteriös bleibt: „Wenn ein gelbrotes Abendlicht zwischen den Stämmen die Augen zum Zwinkern brachte, zuckte der Kopf, machten die Hände entrüstete hastige Abwehrbewegungen“ (Döblin 63). Insgesamt scheint die Natur - anders als bei Heyms Der Irre - für Herrn Fischer die Funktion eines Antipoden zu haben, denn das erblickte Licht provoziert ebenso eine intensive Gegenreaktion wie jene titelgebende Butterblume, der er schließlich den Kopf abschlägt und die er später bemitleidet, was letztlich doch auf eine Verbindung zu ihr hinweist.

Auch bei Fischer findet sich eine ausgeprägte Sensibilität, die offensichtlich zu den angesprochenen Handlungsweisen verleitet. Wie bei Der Irre ist von einer ausgeprägten Wahrnehmungsfähigkeit zu sprechen, die einer Reduktion auf eine Krankheitssymptomatik als Beschreibung von Mängeln entgegensteht. Damit weisen die beiden Texte von Heym und Döblin einen Ansatz auf, der eine Facette von Krankheit aufdeckt, die ihre kranken Protagonisten nicht bloß als Verkörperungen von Krankheit determinieren, sondern Komplementärfähigkeiten aufzeigen und darüber hinaus den Anteil gesellschaftlicher Bedingungen und Prozesse an ihren Dispositionen andenken. Damit werden Aspekte von Krankheit aufgedeckt und es kommt zu einer Enttabuisierung. An den 
Handlungen der beiden Protagonisten lassen sich nicht nur Symptome ablesen, sondern ihr Verhalten zeigt mögliche Entstehungsbedingungen für Krankheit. Es werden Merkmale erkennbar, die psychische Dispositionen nicht allein als Grundlage für Krankheiten erscheinen lassen, sondern auch als potenzielle Befähigungen, die mit Reflexion und Sensibilität einhergehen.

\section{Krankheit als offene Wunde (Franz Kafkas Ein Landarzt)}

Franz Kafkas Erzählung über einen Landarzt entwirft zu Beginn vordergründig klar und eindeutig Konstellation und Szenario der Handlung: Ein Landarzt erhält einen Auftrag und muss sich bei starkem Schneetreiben zu einem Kranken aufmachen, um ihn zu behandeln (vgl. Engelhardt 469-70). So geklärt das Rollenverhältnis zwischen Arzt und Patient erscheinen mag, so wenig wird über die konkrete Erkrankung des Patienten kommuniziert - man erfährt lediglich, es sei „ein Schwerkranker“, der Behandlung erwarte (Kafka 31). Krankheit wird schon von Beginn an durch die lakonische Einführung zu einer Leerstelle des Textes, was sich in der Folge fortsetzt.

Die personale Ich-Perspektive des Arztes, aus der das Geschehen vermittelt wird, und die eingangs explizit gemachte Reise deuten die existenzielle Dimension an, die das Erlebte für den Arzt selbst erhalten wird - die Reise hat weder einen konkreten Beginn noch ein Ende. Dementsprechend antizipiert der Anfang den Schluss der Erzählung und der Arzt wird auf einer nicht endenden Reise präsentiert. Der zu bewerkstelligende Auftrag beginnt mit einer Komplikation, denn das Pferd des Arztes ist zunächst ebenso wenig verfügbar wie ein im Ort geliehenes und schon der erste Satz, „Ich war in großer Verlegenheit“, setzt den Akzent auf eine Problematik, mit welcher sich der Arzt konfrontiert sieht (ebd.). Hier ist also gerade nicht zu erwarten, dass ein Arzt im Rahmen seiner alltäglichen Berufsroutine einen Fall lösen wird.

Die Rätselhaftigkeit des Textes selbst wird durch den Status, den Krankheit darin einnimmt, maßgeblich befördert. Denn auch die erste Untersuchung des Patienten lässt keine Symptome erkennen, die weiterführende Schlüsse zulassen. Und die Einschätzung des Landarztes kulminiert schließlich in dem Verdikt: „der Junge ist gesund, ein wenig schlecht durchblutet, von der sorgenden Mutter mit Kaffee durchtränkt, aber gesund und am besten mit einem Stoß aus dem Bett zu treiben“" (Kafka 34). Diese erste Beurteilung unterliegt schließlich einem Erkenntnisumschwung, der sich wie die Bereitschaft des Arztes zu einer 
anderen Diagnose liest: „tränenvoll in die Lippen beißend und die Schwester ein schwer blutiges Handtuch schwenkend, bin ich irgendwie bereit, unter Umständen zuzugeben, daß der Junge vielleicht doch krank ist“ (Kafka 34-35). Die ostentative Verwendung relativierender Füllwörter bilden den Wandel in der Gesinnung des Arztes deutlich ab. Wenige Sätze später heißt es bereits: „und nun finde ich: ja, der Junge ist krank“ (Kafka 35). Hier setzt der Text fort, was bereits zu Beginn angelegt war: Die Erwartungen an einen Arzt und seine Tätigkeit werden auf die Probe gestellt und enttäuscht, denn der Genese seines Urteils haftet eine erkennbare Kontingenz an.

Schließlich rückt die Wunde des Patienten in den Blick. Ihre detaillierte Beschreibung suggeriert Evidenz auf Basis medizinischer Fachkenntnis: „In seiner rechten Seite, in der Hüftgegend hat sich eine handtellergroße Wunde aufgetan. Rosa, in vielen Schattierungen, dunkel in der Tiefe, hellwerdend zu den Rändern, zartkörnig mit ungleichmäßig sich aufsammelndem Blut, offen wie ein Bergwerk obertags" (Kafka 35). Die sprachliche Genauigkeit weicht mit dem abschließenden Vergleich einer uneigentlichen Ausdrucksweise und eröffnet so einen Raum, der von Assoziation und Interpretation geprägt ist - die diagnostische Betrachtungsweise wird damit von einem anderen Paradigma abgelöst, beides bleibt unverbunden nebeneinander. Und die ärztlichen Ausführungen zur Entstehung der Wunde weisen einen sprachlichen Duktus auf, der mehr zur Allegorie als zum Befund tendiert: „[... ] deine Wunde ist so übel nicht. Im spitzen Winkel mit zwei Hieben der Hacke geschaffen. Viele bieten ihre Seite an und hören kaum die Hacke im Forst, geschweige denn, daß sie ihnen näher kommt“ (Kafka 36). Die Wunde bleibt schließlich „ohne Ursache und ohne pathologisch definierbare Bedeutung" (Kyora 761).

Kafkas Erzählung über einen Landarzt reiht sich in ein Gesamtwerk seines Autors ein, für das Strukturen der Verrätselung programmatisch sind (vgl. Blank 227-28). Dies gilt auch für Krankheit in Ein Landarzt. Rätselhaftigkeit wird gerade durch die Verbindung von Evidenz und ihrer Abwesenheit generiert - was festgestellt wird, wird zugleich zurückgenommen oder unterminiert und was offensichtlich ist, wird durch Kommentierung konterkariert. Dies gilt für die Diagnose des Landarztes wie für die Wunde und der Text wirft so nicht zuletzt auch Fragen zur Urteilsfindung in medizinischen Kontexten auf, wenn er die Perspektive des Landarztes eingehend beleuchtet. In Kafkas Erzählung von einem Landarzt fällt damit Tabuisierung und Enttabuisierung zusammen, denn es ist nicht mehr zwischen Verschlüsselung und Enträtselung zu unter- 
scheiden. Bereits der Beginn des Textes bereitet diesen Rahmen für den ganzen Text vor, wenn er gleichermaßen konkret und nachvollziehbar erscheint, aber doch von Anfang an Komplikationen erwarten lässt und das zu erwartende Geschehen problematisiert.

\section{Körpergrenzen und epistemische Schließungen (David Wagners Leben)}

David Wagners autobiografischer Text Leben steht in einer Reihe von Krankheitsbeschreibungen der Gegenwartsliteratur, die das Spannungsverhältnis zwischen medizinischen Wissensgrundlagen und selbst erlebter Krankheit abbilden (vgl. Reulecke 466-67) und damit auch das Thema Coping reflektieren (vgl. Engelhardt 159-60). Der Textbeginn wird als unauffälliger Verlauf eines Abends inszeniert. Einzig die Kapitelbezeichnung BLUT gibt Anlass zu Skepsis. Durchbrochen wird die Atmosphäre alltäglicher Unscheinbarkeit von einem körperlichen Signal - einem Kratzen im Hals, das beim Zähneputzen von der Gewissheit abgelöst wird, sich übergeben zu müssen: „Ich drehe mich um, beuge mich über die Badewanne, da schwappt es schon aus mir heraus. Als ich die Augen öffne, wundere ich mich über das viele Blut in der Wanne. Langsam läuft es Richtung Abfluß“ (Wagner 9). Auffällig an dieser Schilderung ist die Autonomie körperlicher Vorgänge, die nicht mehr zugehörig zu einem ganzheitlichen Ich wahrgenommen werden, sondern davon entkoppelt und mit einem Eigenleben ausgestattet. Das Ich tritt im Rahmen seiner Beschreibung in Distanz dazu. Hinzu kommt ein Übergang vom Unsichtbaren zum Sichtbaren, denn erst mit dem gesehenen Blut ist die Irritation über das, was vor sich geht, vollständig.

Der Text arbeitet an einer Unmittelbarkeit der Geschehnisse, indem er suggeriert, einer sukzessiven Abfolge von Ereignissen beizuwohnen. Die ostentative Plötzlichkeit der Vorgänge vermittelt Lesenden den Eindruck, dem beschriebenen Geschehen selbst beizuwohnen. Damit verschleiert der Text seinen Status als retrospektiv niedergeschriebenes Dokument, an dessen Verschriftlichung Erinnerung wesentlich beteiligt ist. Erst der eingeschobene Rekurs auf medizinisches Wissen zu den ablaufenden Vorgängen und ihren Hintergründen löst die Perspektive auf das Geschehen auf und signalisiert in seiner Ausführlichkeit, dass die zeitliche Dimension des unmittelbar Erlebten verlassen wurde. Dieser epistemische Einschub fungiert zudem als Gegengewicht zu der chaotischen und plötzlich eintretenden Situation und impliziert Entschleunigung des Erzähltempos wie auch eine Rationalisierung der Vorgänge - obwohl das geschilderte Ereignis unerwartet eintritt, schafft der Verweis auf den Inhalt des 
Arzt-Patienten-Austauschs eine Folie, die den Ablauf einordnet und das Geschehen vom Unerwarteten in das Erwartbare umwandelt:

Ich weiß, was das bedeutet. B., mein Arzt, der mich seit meinem zwölften Lebensjahr behandelt, hat mich oft genug, seit Jahren schon, gewarnt. Ich weiß, daß die Ösophagusvarizen, die Krampfadern in meiner Speiseröhre, geplatzt sind, ich weiß, daß ich nun nach innen blute und nicht ohnmächtig werden darf, ich muß den Notarzt rufen (Wagner 10).

Insgesamt dreimal werden formelhaft Ausführungen mit „Ich weiß“ eingeleitet. Es handelt sich um einen sprachlichen Akt der Selbstvergewisserung, der einem Mantra gleicht und Sicherheit in einer unsicheren Situation schaffen soll. Dieses Wissen wird in der Folge aber auch zu einem Kontrapunkt zu den Eindrücken des eintreffenden medizinischen Personals:

Ich begegne dem Notarzt und den beiden Rettungssanitätern im Treppenhaus, grüße und sage: Ich bin's, ich muß in die Klinik, und merke gleich, sie halten mich für einen Simulanten, sie haben die Badewanne nicht gesehen. Im Krankenwagen, ich sitze auf dem Transportstuhl, Rücken in Fahrtrichtung, weiß der Arzt nichts mit mir anzufangen, er sieht sich meinen Notfall- und den Organspendeausweis an. (Wagner 10-11)

Die Komik der Situation kommt auch durch das Gefälle von wissendem Patienten und unwissendem Fachpersonal zustande. Das Blut in der Badewanne erfährt eine Bedeutungssteigerung und wird zum Symbol erhoben, das auch mit dem Titel Leben eng verknüpft ist - im textlichen Szenario steht es für Evidenz, die eine Verbindung zu Krankheit bestätigt.

Krankheit tritt zu Beginn des Textes also vor allem durch den körperlichen Austritt von Blut in Erscheinung und ruft ein Wissen über Krankheit auf. Doch erst die Sichtbarkeit veranlasst dazu, die erkennbaren Indizien in diesem $\mathrm{Zu}$ sammenhang zu verorten, wie durch das Verhalten des medizinischen Personals ersichtlich wird: Die Entäußerung des eigenen Blutes löst Verwunderung aus und bringt das Verborgene in einem körperlichen Akt zur Sichtbarkeit. Dieser Austritt des Blutes über die Grenze des Körpers nach draußen wird als Vorgang in Szene gesetzt, der zunächst als Tabu erscheinen mag, wenn die Grenzen normalen menschlichen und gesunden Lebens überschritten werden. Tatsächlich schließt sich an die Rätselhaftigkeit der ersten körperlichen Symptome ein Prozess der Klärung an, der den Vorgängen ihre Rätselhaftigkeit nimmt und sie 
in einer Wissensmatrix verortet, die auf medizinischem Wissen und ärztlicher Beratung fußt. Der Schrecken weicht schließlich dem Verstehen und die Anteile des Tabuisierten werden aufgelöst durch einen rationalen Umgang mit den Abläufen, der durch das eigene Wissen ermöglicht wird.

\section{8. (Aus-)gesetzte Krankheit (Johann Wolfgang von Goethes Erlkönig)}

Von Anfängen zu sprechen, impliziert grundsätzlich eine strukturelle Organisation literarischer Texte, die aufeinanderfolgende Sequenzen vorsieht und damit auch eine Zeitlichkeit von Ereignissen, die in einer Handlung aufgeht. Gerade bei lyrischen Texten wird sich dieses Charakteristikum vielfach nicht finden lassen, bei dramatischen Texten und Erzählungen in ihren unterschiedlichen Untergattungen hingegen schon. Bei Johann Wolfgang von Goethes Erlkönig als Beispiel für eine Ballade, die bekanntermaßen auch als Handlungsgedicht definiert werden kann, ergibt sich eine Chronologie der Abläufe und der Text zeichnet sich durch einen typisch dramatischen Aufbau der Handlung auf. Dementsprechend kann gerade für diesen Text auch die Frage nach einem Anfang, der mit Krankheit zusammenfällt, produktiv gemacht werden.

Bei den bisher behandelten Beispielen wurde Krankheit als Phänomen behandelt, das Gültigkeit für die Diegese beansprucht und insofern als Fakt innerhalb der Fiktion anzusehen ist. Im Falle des Erlkönig-Textes drängt es sich geradezu auf, diese implizit vorgenommene Setzung zu hinterfragen und zu problematisieren: Stephan Mühr arbeitet sich kritisch am Deutungskanon zu Goethes Ballade ab und macht dabei anschaulich, wie selbstverständlich Krankheit zu einem integrativen Bestandteil des Textes gemacht und dadurch eine traditionelle Lesart etabliert wird (Mühr 197-98). Er zeigt damit auch, dass Krankheit nicht nur eine Basis für Interpretationsspielräume darstellt, sondern die Konsequenz einer Setzung von Krankheit im Rahmen der Textinterpretation auch Einschränkungen hervorbringt, die ästhetische Potenziale eines Textes einschränken können.

Krankheit als Erklärungsmuster, das die Textinterpretation von Erlkönig stützt, folgt Prinzipien, die auf Rationalität gegründet sind. Dies mag für interpretatorische Bestrebungen einleuchten, da Interpretationen durch ihre Plausibilität erst intersubjektive Überzeugungskraft entfalten können. Eine rationalisierte Perspektive auf den Text führt jedoch zu Schwerpunktsetzungen, die sich reduktionistisch auswirken: Der ersten Erwähnung des Erlkönigs durch 
den Sohn geht die Frage des Vaters nach dem Verhalten des Sohnes voraus, der sich zu verbergen trachtet. Der Dialog von Vater und Sohn folgt dann einer Rollenverteilung, bei der die väterliche Intention, den unruhigen Sohn zu beruhigen, hervortritt. Deshalb nimmt er die Rolle desjenigen ein, der rationale Erklärungen für undurchsichtige und rätselhafte Vorgänge zu liefern vermag. Diese Sichtweise schließt die Existenz einer Gestalt wie der des Erlkönigs kategorisch aus und damit ebenso von ihr ausgehende Wirkungen, die den Tod des Sohnes hervorrufen - eine Alternative bestünde darin, Krankheit als Effekt des vom Sohn Erlebten zu begreifen, doch auch dann wäre die Kopplung von Wahrnehmung und Krankheit nicht aufgelöst und käme einer Pathologisierung gleich. Denn der väterlichen Orientierung an der Vernunft als verstandesgemäße und gesunde Perspektive wäre die phantastische und dem gesunden Menschenverstand entbehrende Wahrnehmung des Sohnes dichotomisch gegenübergestellt, ohne einen Einbruch des Anormalen und Irrationalen in die Realität als Möglichkeit anzuerkennen. Insofern entspricht Krankheit als Deutungsmuster für den Text einer Positionierung, die mit dem Vater kongruiert und mit dem Sohn divergiert.

Dies hat auch Auswirkungen auf die Lesart des Textanfangs: Der Vater schützt seinen Sohn behutsam vor Krankheit, ob diese ihm durch den Ritt erst noch droht oder ob dem Vater bereits eine Erkrankung des Sohnes bekannt ist der Text gibt hierauf keine konkrete Antwort. Doch diese Lesart tendiert dazu, den Sohn unter einen pathologisierenden Generalverdacht zu stellen und ihn mitsamt seiner Wahrnehmung zu marginalisieren. Dabei ist eine Auffälligkeit an der Textkonzeption zu berücksichtigen, die eine ungeklärte Frage birgt: Wer vermag eigentlich zu vernehmen, was der Erlkönig spricht? Offensichtlich ist dies für den Sohn und ebenso für Lesende der Fall. Prinzipiell ist nicht darauf zu schließen, dass der Junge die Worte des Erlkönigs übermittelt, denn sie sind formal und sprachlich in keinen Vermittlungszusammenhang eingebettet und werden kommentarlos in direkter Rede wiedergegeben - textlich kommt ihnen damit ein eigenständiger und isolierter Status zu, der sie wiederum vom Jungen entkoppelt, selbst wenn dieser auch in der Lage sein sollte, die Worte des Erlkönigs zu perzipieren.

Die Worte des Erlkönigs erhalten also ein Eigengewicht und stehen zwischen Vater und Sohn. Es wäre daher ein Zugeständnis, sie lediglich dem Sohn und seiner Wahrnehmung zuzuordnen, und zugleich eine Tabuisierung. Denn Krankheit wird im beschriebenen Deutungszusammenhang eine interpretato- 
rische Funktion zugewiesen, welche die Wahrnehmung des Sohnes plausibilisiert und zugleich entwertet. Krankheit fungiert als Tabu, denn das Übernatürliche wird als Element einer pathologischen Wahrnehmung gedeutet und die Interpretation schlägt sich dann auf die Seite jener Erklärbarkeit, die dem rational argumentierenden Vater entspricht - indem spezifischen Textelementen, die mit Krankheit assoziiert werden, die Legitimität abgesprochen wird, kommt es zu einer Auflösung jenes Schwebezustandes und schließlich zu einer Übertragung des Tabus auf die Sphäre des Phantastischen: Der Phantast ist zugleich der Kranke und sein Blick auf die Realität entspricht nicht der gesunden Norm. Insofern wird Krankheit in diesem prominenten Beispiel der Literaturgeschichte $\mathrm{zu}$ einem Katalysator für Tabuisierung und weniger selbst zum zentralen Gegenstand einer Tabuisierung oder Enttabuisierung. Goethes Text steht dabei stellvertretend für eine Vielzahl romantischer Textkonzeptionen, die ähnliche Dispositionen aufweisen. Zu denken wäre exemplarisch an E. T. A. Hoffmanns Sandmann, der mit der Setzung von Krankheit als Interpretationsgrundlage die Pathologisierung von Wahrnehmung gleichermaßen werden lässt. Eine entsprechende gedankliche Linie wäre bis zu Franz Kafkas Texten zu ziehen und dies insbesondere für psychoanalytisch grundierte Ausdeutungen (vgl. Höfle 114-15).

\section{Fazit}

Im vorliegenden Beitrag wurde ein Panorama an Textbeispielen betrachtet. Damit wurde die literarische Vielschichtigkeit im Umgang mit dem Thema Krankheit ebenso deutlich wie der variierende Status von Krankheit und ihren Funktionen. Nicht zu verleugnen ist ein Schwerpunkt der Textauswahl auf der Literatur um 1900, deren Auseinandersetzung mit Krankheit nicht nur wegen der Quantität signifikanter Textbeispiele relevant ist, sondern auch wegen der Diversität behandelter Phänomene. Dabei wäre noch konkreter herauszuarbeiten, wie die Literatur um 1900 gerade aufgrund ihrer ästhetischen Verfahren für die auf sie folgende Literaturgeschichte bis einschließlich zur Gegenwartsliteratur maßgeblich ist. Dass die Literatur der Wende vom zwanzigsten zum einundzwanzigsten Jahrhundert Krankheit häufig als Gegenentwurf zu bürgerlich-gesellschaftlicher Normalität entwirft, darf jedoch als historisches Spezifikum gelten, das repräsentativen Niederschlag im Konzept der décadence (vgl. Kafitz) findet und insbesondere in Thomas Manns Frühwerk präsent ist, aber auch noch Spuren im 1947 veröffentlichten Doktor Faustus hinterlassen hat. Für die 
Gegenwartsliteratur erscheint hingegen weniger geklärt, was ,die Gesellschaft eigentlich ist - daraus ergibt sich reziprok eine andere Basis für die Betrachtung von Krankheit und es ist zu vermuten, dass Krankheit weniger mit Funktionen belegt, sondern der Versuch unternommen wird, sie nachzuvollziehen und mit ihrer Authentizität nach einem Phänomen zu fragen, das beispielsweise auch für Autorinszenierungen in der Gegenwart Hochkonjunktur hat.

Pauschal von einer Enttabuisierung von Krankheit in literarischen Texten zu sprechen, wird den in diesem Beitrag dargestellten Erkenntnissen nicht gerecht. Vielmehr offenbart Krankheit in literarischen Texten eine reziproke kommunikative Situation zwischen Tabuisierung und Enttabuisierung. Zugegebenermaßen wird Krankheit angesprochen, reflektiert und damit auch sichtbar gemacht und es werden beispielsweise Einblicke in Spezifika des Arztberufes beleuchtet, die außerhalb von Literatur weitgehend unterbelichtet bleiben. Aber Krankheit haftet ein unauflösbarer Rest an, der sie mit Tabus verschränkt sein lässt - insofern ist es gerade eine Leistung literarischer Texte, ein ganzheitliches Bild von Krankheiten zu konstruieren, das einen rationalen Zugriff ermöglicht, aber sich diesem zugleich entzieht und psychische wie emotionale Aspekte von Krankheit mitbedenkt. Schließlich wäre dem aufklärerischen Bestreben einer lückenlosen Erklärbarkeit von Krankheit ein Moment zu eigen, das den Menschen und die Konsequenzen verleugnet, mit denen er sich durch Krankheit konfrontiert sieht.

Auffällig ist, dass nicht nur Krankheit partiell tabuisiert oder enttabuisiert wird, sondern Krankheit funktional an Tabus und ihrer Auflösung beteiligt ist, wie an Thomas Manns Der Tod in Venedig oder Georg Heyms Der Irre ersichtlich wurde. Gezeigt hat sich zudem, dass Krankheit bei Literatur vielfach mit Setzungsvorgängen verknüpft ist - damit ist nicht in erster Linie gemeint, wie Texte selbst durch Schlagworte und Informationen auf die Präsenz von Krankheit hinweisen, sondern dass literarische Texte als kommunikative Geflechte fungieren, auf deren Grundlage Lesende Schlüsse ziehen und Setzungen als Teil einer Interpretations- und Decodierungspraxis vornehmen. Die Mehrdeutigkeit des literarischen Textes eröffnet eine Betrachtung von Indizien, die als Symptome gelesen werden können - aber nicht immer geht damit eine verbindliche Erklärung einher, mit der abschließend verifiziert werden könnte, dass Krankheit Thema oder Motiv eines Textes ist. Indem ein Zustand des Übergangs bewahrt bleibt, der offen lässt, ob dezidiert auf ein Krankheitsbild geschlossen werden kann, verweigert Literatur einer Grenzziehung, die basierend auf Dichotomien 
des Gesunden und Normalen oder Kranken und Anormalen eine Klassifizierung und Zuordnung perpetuiert. Auch wenn Literatur Krankheit thematisiert, fungiert der Text immer noch als Raum der Aushandlung und setzt damit ein Gegengewicht zu sozialen Ordnungen, die abseits davon Gültigkeit beanspruchen.

Der konzentrierte Blick auf Textanfänge hat gezeigt, dass wesentliche Charakteristiken der Inszenierung von Krankheit bei Literatur schon zu Beginn präfiguriert werden. So geben erste Konstellationen und Rollendarstellungen wie in Kafkas Ein Landarzt Aufschluss darüber, was davon zu erwarten ist, wie ein Text in der Folge mit Krankheit umgehen wird. Für David Wagners Leben ist hingegen gerade die Verwendung von starken Kontrasten zentral, wenn mit Krankheit verbundene Vorgänge zunächst undurchsichtig erscheinen und schließlich doch souverän erklärt werden - eine Erwartung Lesender an Krankheit wird vorbereitet und insofern ein literarisches Spiel initiiert, das Teil einer ästhetischen Programmatik im Umgang mit Krankheit und Wissen sowie Unwissen darüber ist.

\section{Literaturverzeichnis}

\section{Primärliteratur}

Benn, Gottfried. "Gehirne". Gottfried Benn. Prosa und Autobiographie in der Fassung der Erstdrucke. Bruno Hillebrand (Hg.). S. Fischer, 2006, S. 19-23.

Büchner, Georg. "Lenz". Georg Büchner. Werke und Briefe. Karl Pörnbacher et al. (Hg.). dtv, 2006, S. 135-58.

Döblin, Alfred. "Die Ermordung einer Butterblume”. Alfred Döblin. Die Ermordung einer Butterblume und andere Erzählungen. Christina Althen (Hg.). dtv, 2010, S. 63-77.

Hesse, Hermann. "Stufen”. Das erzählerische Werk. Sämtliche Jugendschriften, Romane, Erzählungen, Märchen und Gedichte. Bd 10. Die Gedichte. Volker Michels (Hg.). Suhrkamp, 2012, S. 366.

Heym, Georg. "Der Irre”. Georg Heym. Das Werk. Monika Weißenberger (Hg.). Zweitausendeins, 2005, S. 18-28.

Kafka, Franz. Das Urteil und andere Erzählungen. Peter Höfle (Hg.). Suhrkamp, 2003, S. 31-37.

Mann, Thomas. “Der kleine Herr Friedemann”. Die Erzählungen. S. Fischer, 2005, S. 66-94.

---. Der Tod in Venedig. S. Fischer, 2007.

Röder, Hilda. Henning flieht vor dem Vergessen Ein Roman über Würde, Alzheimer-Erkrankung und Sterbehilfe. Printsystem-Medienverlag, 2013. 


\section{Sekundärliteratur}

Alt, Peter-André. „Jemand musste Josef K. verleumdet haben“. Erste Sätze der Weltliteratur und was sie uns verraten. C. H. Beck, 2020.

Blank, Juliane. "Ein Landarzt. Kleine Erzählungen”. Kafka-Handbuch. Leben - Werk - Wirkung. Manfred/ Engel und Bernd Auerochs (Hg.): Metzler, 2010, S. 218-40.

Clare, Jennifer et al. (Hg.): Schreibprozesse im Zwischenraum. Zur Ästhetik von Textbewegungen. Winter, 2018.

Engelhardt, Dietrich von. „Coping“. Literatur und Medizin. Ein Lexikon. Bettina von Jagow und Florian Steger (Hg.). Vandenhoeck \& Ruprecht, 2005, S. 159-63.

---. „Landarzt“. Literatur und Medizin. Ein Lexikon. Bettina von Jagow und Florian Steger (Hg.). Vandenhoeck \& Ruprecht, 2005, S. 469-74.

Engelns, Markus et al. (Hg.). Schlusspunkt. Poetiken des Endes. Königshausen \& Neumann, 2017.

Erhart, Walter. „Einleitung“. Der Deutschunterricht 5, 2003, S. 2-6.

Foucault, Michel. Die Ordnung des Diskurses. S. Fischer, 1993.

Glasenapp, Nicolai. "Lebt man in Vergessenheit? Zum Konnex von Sterben, Tod und Demenz in deutschsprachiger Gegenwartsliteratur." LIMBUS - Australisches Jahrbuch für germanistische Literatur- und Kulturwissenschaft Bd. 8, 2015, S. 147-65.

Habermann, Frank. Literatur/Theorie der Unsagbarkeit. Ergon, 2012.

Höfle, Peter. „Kafka und sein Publikum - Interpretationslinien“. Das Urteil und andere Erzählungen. Peter Höfle (Hg.). Suhrkamp, 2003, S.111-17.

Kafitz, Dieter: Décadence in Deutschland. Studien zu einem versunkenen Diskurs der 90er Jahre des 19. Jahrhunderts. Winter, 2004.

Kyora, Sabine. „Symptom. „. Literatur und Medizin. Ein Lexikon. Bettina von Jagow und Florian Steger (Hg.). Vandenhoeck \& Ruprecht, 2005, S. 758-62.

---. „Zwang“. Literatur und Medizin. Ein Lexikon. Bettina von Jagow und Florian Steger (Hg.). Vandenhoeck \& Ruprecht, 2005, S. 868-72.

Martínez, Matías und Michael Scheffel. Einführung in die Erzähltheorie. C. H. Beck, 2016.

Mühr, Stephan. „Der Sohn im „Erlkönig“ hat kein Fieber“. Magie und Sprache. Carlotta von Maltzan (Hg.). Peter Lang, 2012, S. 197-209.

Müller-Tamm, Jutta. „Schwindel“. Literatur und Medizin. Ein Lexikon. Bettina von Jagow und Florian Steger (Hg.). Vandenhoeck \& Ruprecht, 2005, S. 704-08.

Reents, Friederike. „Gehirne. Novellen (1916): Rönne-Novellen.“ Benn-Handbuch. Leben Werk - Wirkung. Christian M. Hanna (Hg.). Metzler, 2016, S. 130-36.

Reidy, Julian. Raum und Interieurs in Thomas Manns Erzählwerk. Materielle Kultur zwischen ,Welthäusern' und ,Urdingen' de Gruyter, 2018.

Reulecke, Anne-Kathrin. „Neue Pathographien. Transplantation als Grenzerfahrung in David Wagners ,Leben“". Zeitschrift für Germanistik, H. 28, Nr. 3., 2018, S. 465-85.

Schwahl, Markus und Christian Thienel. „Zwischen Empirie und Metapher. Zur literarischen Domestizierung von Krankheit in Thomas Melles Die Welt im Rücken und 
Hermann Kinders Der Weg allen Fleisches". Texte der Gegenwartsliteratur und Perspektiven für den Literaturunterricht. Jan Standke und Dieter Wrobel (Hg.). Wissenschaftlicher Verlag, 2019, S. 261-84.

Schünemann, Peter. Georg Heym. Morgenbuch, 1993.

Shattuck, Roger. Tabu. Eine Kulturgeschichte des verbotenen Wissens. Aus dem Amerikanischen von Harald Stadler und Thorsten Schmidt. Piper, 2000.

Shi, Lingzi. Nervenliteratur und nervöse Gesellschaft. Krankheitsdiskurse in Thomas Manns früher Prosa. Verlag Dr. Kovač, 2019.

Stiening, Gideon. Literatur und Wissen im Werk Georg Büchners. Studien zu seinen wissenschaftlichen, politischen und literarischen Texten. de Gruyter, 2019.

Trumm, Tanja. Dem Schweigen Worte geben. Wege der Annäherung an Tabu und Tabuisierung im Deutschunterricht. Schneider Verlag, 2014. 


\title{
LITERARY ANAMNESES. ILLNESSES AND THEIR TEXTUAL BEGINNINGS
}

\begin{abstract}
Nicolai GLASENAPP

Universität Koblenz-Landau, Germany

Universitätsstraße 1, 56070 Koblenz

nglasenapp@uni-koblenz.de
\end{abstract}

Literary texts present illnesses as parts of a communicative structure that constitutes illness itself. It remains to prove whether literature takes part in a process that can be described as removal of illness-related taboos or if the specific figuration of literary texts tends to partly relate to the process of tabooing. This paper analyses literary texts by Georg Büchner, Thomas Mann, Gottfried Benn, Franz Kafka, Georg Heym, Alfred Döblin, and David Wagner, and considers their beginnings as a context for the introduction of illness. Special focus is given to what is said and what is unsaid in this context, indicating questions of what is (un)known about illness. The paper focuses on central categories connected with the configuration of illnesses and their textual beginnings: symbolic spaces; causality and destiny; action as illness; illness as an open wound; borders of the body and epistemic closures; setting and suspension of illness as part of interpretation. As a central result, the paper points out that illness does not detach from taboo completely but that it oscillates between tabooing and removal of taboos. In this way, literature tries to present illness as a whole and avoids exaggerating a rational perspective on illness that suppresses important aspects of human subjects, which has massive implications for the linked topics literature, knowledge and illness.

Keywords: illness, (removal of) taboo, Georg Büchner, Thomas Mann, Gottfried Benn, Franz Kafka, Georg Heym, Alfred Döblin, David Wagner, wound, borders of the body 


\section{KNJIŽEVNE ANAMNEZE. BOLESTI I NJIHOVI TEKSTUALNI POČECI}

\begin{tabular}{c} 
Sažetak \\
\hline Nicolai GLASENAPP \\
Universität Koblenz-Landau, Njemačka \\
Universitätsstraße 1,56070 Koblenz \\
nglasenapp@uni-koblenz.de
\end{tabular}

Književni tekstovi prikazuju bolesti kao dijelove komunikacijske strukture koja tvori samu bolest, pri čemu je glavno pitanje sudjeluje li književnost u procesu koji je moguće opisati kao otklanjanje tabua povezanih s bolešću ili određena tvorba književnih tekstova djelomično sudjeluje u procesu tabuiziranja. Rad analizira književne tekstove Georga Büchnera, Thomasa Manna, Gottfrieda Benna, Franza Kafke, Georga Heyma, Alfreda Döblina i Davida Wagnera u kontekstu njihovih početaka kao uvod u temu bolesti. Posebna pozornost usmjerava se na ono što je izrečeno, kao i na ono što nije, što ukazuje na pitanja poznatog i nepoznatog o bolesti. Rad se usredotočuje na središnje kategorije povezane s tvorbom bolesti i njihovim tekstualnim počecima: simboličkim prostorima, uzročnost i sudbinu, djelovanje kao bolest, bolest kao otvorena rana, granice tijela i epistemsko zatvaranje, mjesto i suspenzija bolesti kao dio tumačenja. U konačnici, rad ističe da se bolest ne odvaja od tabua u potpunosti, nego da se koleba između tabuiziranja i otklanjanja tabua. $\mathrm{Na}$ taj način književnost pokušava prikazati bolest u cjelini i izbjegava pretjeranu racionalnu perspektivu bolesti koja potiskuje važne aspekte ljudskih subjekata, što se uvelike odražava na povezane teme književnosti, znanja i bolesti.

Ključne riječi: bolest, tabu, otklanjanje tabua, Georg Büchner, Thomas Mann, Gottfried Benn, Franz Kafka, Georg Heym, Alfred Döblin, David Wagner, uzročnost i sudbina, rana, tjelesne granice 